\title{
ORIGINAL ARTICLE THE RELATIONSHIP BETWEEN EPICARDIAL FAT TISSUE THICKNESS AND RISK OF FREQUENT PREMATURE VENTRICULAR CONTRACTIONS
}

\author{
Seyedeh Fatemeh Mirrazeghi ${ }^{1}$, Shadi Shabahang ${ }^{1}$, Azin Vakilpour ${ }^{1}$, Arsalan Salari ${ }^{1}$, Soheil \\ Hassanipour ${ }^{1}$, Mahboobeh Gholipour ${ }^{1}$ \\ ${ }^{1}$ Cardiovascular Diseases Research Center, Department of Cardiology, Heshmat Hospital School of Medicine, Guilan University of \\ Medical Sciences, Rasht, Iran
}

\begin{abstract}
Objectives: This study aimed to evaluate the relation between epicardial fat tissue (EFT) thickness and frequent premature ventricular contractions.

Methodology: In this cross-sectional study, patients with no structural heart diseases diagnosed with PVCs on a 24 holter rhythm monitoring were included. EFT thickness was measured by 2-dimensional transthoracic echocardiography. PVCs of more than 10 per hour were considered as frequent PVCs. To investigate the effect of independent variables, univariate logistic regression was performed.

Results: Of total 50 studied patients, $64 \%$ were females. The mean age of subjects was $46.8 \pm$ 13.1 years. Twenty-five patients were experiencing frequent PVCs of $>10$ per hour. In univariate analysis, age $(\mathrm{OR}=1.05,95 \% \mathrm{CI} ; 1.01-1.10, \mathrm{p}=0.050)$, left ventricular end diastolic diameter (LVEDD) $(\mathrm{OR}=1.14,95 \% \mathrm{CI} ; 1.01-1.28, \mathrm{p}=0.036)$ and left atrial (LA) diameter $(\mathrm{OR}=1.35,95 \% \mathrm{CI} ; 1.11-1.62, \mathrm{p}=0.002)$ were significantly associated with developing frequent PVC. EFT thickness was positively correlated with age $(r=0.389, p=0.005)$ and interventricular septum thickness in diastole (IVSD) $(r=0.384, \mathrm{p}=0.006)$. No significant correlation between EFT and PVCs was found ( $\mathrm{p}=0.669)$.

Conclusion: Patients with frequent PVCs had significantly higher LVEDD and LA diameter. Although patients with higher EFT thickness were more likely to experience frequent PVCs, there was no statistically significant correlation between EFT thickness and frequent PVCs.

Keywords: arrhythmia, epicardial fat tissue, premature ventricular contractions
\end{abstract}

Citation: Mirrazeghi SF, Shabahang S, Vakilpour A, Salari A, Hassanipour S, Gholipour M. The Relationship between Epicardial Fat Tissue Thickness and Risk of Frequent Premature Ventricular Contractions. Pak Heart J. 2021;54(03):249-253. DOI: https://doi.org/10.47144/phj.v54i3.2112

\section{INTRODUCTION}

Premature ventricular contractions (PVCs) are one of the most common arrhythmias, with the reported prevalence of about 40 to $75 \%$ in general population on 24-48 hour holter monitoring and between 1 to $4 \%$ in patients detected by 12-lead electrocardiography. ${ }^{1,2}$ PVC is an early depolarization arising from the ventricles and is characterized by premature QRS complexes that appear wide (usually $>120 \mathrm{mseq}$ ) and are not preceded by a $\mathrm{p}$ wave in electrocardiogram. ${ }^{3,4}$ The majority of patients are asymptomatic, but PVCs are a common cause of a variety of symptoms such as palpitation, feeling of pause in cardiac beats, dizziness and rarely ventricular tachycardia accompanied by shortness of breath, chest discomfort, hypotension or/and syncope. ${ }^{3} \mathrm{PVC}$ in individuals with no history of structural heart diseases is relatively common and has been thought to be a benign phenomenon for years. Meanwhile, recent literature have revealed that recurrent PVCs may be associated with the occurrence of myocardial infarction, developing ventricular fibrillation (VF), and mortality in healthy subjects. ${ }^{3,5}$
The amounts of PVC attacks of more than 10-25\% of total heart beats in a day have been reported to be associated with decreased ventricular function, ventricular dilation, and increased risk of cardiomyopathy. ${ }^{2,6}$ Related risk factors and etiology of $\mathrm{PVC}$ are not fully known in patients with normal heart structure. However, some cardiac disorders such as ischemia, myocardial hypertrophy, cardiomyopathy and chronic heart failure have been reported to be potential predisposing factors in developing frequent PVCs in patients suffering from structural heart diseases. ${ }^{1,7}$ PVCs are often associated with structural heart diseases and can result in some cardiovascular effects including ventricular systolic and diastolic dysfunction as well as decreased ventricular capacity and also can deteriorate existing cardiomyopathy, in which case, PVC suppression may only make a relative improvement in left ventricular dysfunction. ${ }^{1,2}$

Epicardial fat tissue (EFT) is a type of visceral fat which surrounds the heart and is used to measure visceral adiposity. There is a close anatomical and functional relationship between EFT and 
myocardium. ${ }^{6,8}$ EFT has been a topic of interest lately because of its detrimental effects on the cardiovascular system due to its role in inflammation, insulin resistance, high levels of free fatty acids and beta-adrenergic activity. ${ }^{9}$ As mentioned in the previous literature, an association between EFT and structural and ultrastructural cardiac changes such as left ventricular and atrium enlargement, atherosclerosis, hypertension, diastolic dysfunction as well as atrial fibrillation has been found. ${ }^{10}$ The relationship between EFT thickness and PVCs has never been established, and until recently, there has been no reliable evidence about this issue. Nevertheless, a prior study indicated a notable relationship between increased EFT thickness and post catheter ablation recurrent ventricular tachycardia associated with structural heart diseases. ${ }^{11}$

Some potential mechanisms to explain the relationship of PVCs and EFT are as follows: atrial and ventricular enlargement which are associated with epicardial fat thickening may predispose the heart to arrhythmias as a result of increased fibrosis and electrophysiological changes. ${ }^{12}$ Also, EFT is a kind of lipid storage which has a role in local and systemic inflammation via releasing inflammatory cytokines (e.g. TNF- $\alpha$, IL1 and IL6) and chemokines that result in cardiac ultrastructural changes including myocardial fibrosis. ${ }^{13}$ An experimental study depicted that these changes can lead to alterations in action potentials in the myocardium and can facilitate the development of PVCs. ${ }^{14}$

Based on the mentioned hypothesis and lack of adequate information in evaluating the independent association between EFT with PVC, the major objective of this study was to investigate the potential relationship of EFT and occurrence of frequent PVC in patients with no structural heart diseases in order to determine the role of EFT thickness as a potential mechanism in the pathogenesis of PVCs.

\section{METHODOLOGY}

This cross sectional study was performed on 50 outpatients referring to the clinic of Dr. Heshmat hospital, the only specialized center of cardiovascular diseases in Rasht, Iran, between May to November 2019.

The study protocol was in accordance with the guidelines of the 2013 version of the Helsinki Declaration and was approved by the ethics committee of Guilan University of Medical Sciences (Research No. IR.GUMS.REC.1398.275)
A total of 50 consecutive patients without structural heart diseases with the complaints of palpitation or feeling a pause in heart beats who were diagnosed with PVC by a $24 \mathrm{~h}$ rhythm holter monitoring were recruited.

Frequent PVC was defined as a repetition of more than 10 times per hour detected by $24 \mathrm{~h}$ rhythm holter monitoring. Exclusion criteria were as follows: having structural heart diseases including heart failure, dilated or hypertrophic cardiomyopathy, coronary artery diseases (previous myocardial infarction, coronary artery bypass graft (CABG), percutaneous coronary intervention (PCI) and angina pectoris), valvular diseases, use of antiarrhythmic drugs, poor echocardiographic imaging, positive exercise test and reluctance to participate in the study. After explaining the purpose of the inquiry and obtaining written consent, demographic and clinical information such as age, sex, history of diabetes, hypertension, hyperlipidemia, and smoking status were obtained from all study subjects via face to face interviews by a researcher of the team. Body weight was measured to the nearest $0.5 \mathrm{~kg}$ and height to the nearest $0.1 \mathrm{~cm}$ using a Seca 755 dial column. Thereafter, body mass index (BMI) was calculated by dividing weight in kilograms by height in meters squared $\left(B M I=k g / \mathrm{m}^{2}\right)$. A standard 12 leads electrocardiogram was obtained from all study subjects by a sophisticated and trained nurse.

Transthoracic echocardiography was performed on the entire study population by a cardiologist who was unaware of patients' clinical features, using 2D echo of Siemens device. Parasternal and apical views (2chamber, 4chamber, and long axis) were measured by Siemens SC2000 echocardiography device. Left ventricular end diastolic and systolic diameters, interventricular septum, left atrium diameter, and left ventricular ejection fraction assessment were accomplished using 2D method.

Epicardial fat thickness identified as echo free space in front of the right ventricular free wall in long axis parasternal view at the end of systole and perpendicular to right ventricular free wall at annulus of the aortic valve (as anatomic reference), was measured in millimeters in three consecutive cardiac cycles. The mean obtained was considered as the thickness of the pericardial fat.

Continuous variables are presented as mean and standard deviation (SD), while categorical variables are shown as absolute numbers and frequency (percentage). 
Independent t-test, Chi square or Fisher exact test and Pearson correlation test were used to analyze the data. In order to investigate the effect of independent variables, univariate logistic regression was performed. Data were analyzed using SPSS (Version 24) software. P-value of $\leq 0.05$ was considered statistically significant.

\section{RESULTS}

Out of 50 studied subjects, 25 patients were experiencing frequent PVC of $>10$ per hour, and 25 patients had PVC repetition of $<10$ per hour in a $24 \mathrm{~h}$ holter monitoring. Of total, $64 \%$ were females and the mean age of subjects was $46.8 \pm 13.1$ years. The mean of BMI was $28.1 \pm 4.9 \mathrm{~kg} / \mathrm{m}^{2}$.

Co-morbidities related to developing frequent PVC are presented in table 1. According to the results, there was no statistical difference between diabetes, hypertension, hyperlipidemia, and smoking with risk of developing frequent PVC ( $\mathrm{p}>0.05)$.

Table 1: Co-morbidities related to developing frequent $\mathrm{PVC}$

\begin{tabular}{|l|c|c|c|}
\hline Risk factor & $\begin{array}{c}\text { PVC < 10 } \\
(\mathbf{N = 2 5})\end{array}$ & $\begin{array}{c}\text { PVC > 10 } \\
(\mathbf{N = 2 5 )}\end{array}$ & P-value \\
\hline Diabetes & $5(20 \%)$ & $6(24 \%)$ & 0.733 \\
\hline Hypertension & $8(32 \%)$ & $11(44 \%)$ & 0.382 \\
\hline Hyperlipidemia & $9(36 \%)$ & $9(36 \%)$ & 0.999 \\
\hline Smoking & $0(0 \%)$ & $1(4 \%)$ & 0.312 \\
\hline
\end{tabular}

$P V C=$ premature ventricular contraction

Demographic and echocardiographic parameters were assessed by univariate logistic regression analysis. In univariate analysis, age $(\mathrm{OR}=1.05,95$ $\% \mathrm{CI} ; 1.01-1.10, \mathrm{P}=0.050)$, left ventricular end diastolic diameter $(\mathrm{LVEDD})(\mathrm{OR}=1.14,95 \% \mathrm{CI}$; 1.01-1.28, $\mathrm{P}=0.036)$ and left atrial (LA) diameter $(\mathrm{OR}=1.35,95 \% \mathrm{CI} ; 1.11-1.62, \mathrm{P}=0.002)$ were significantly associated with developing frequent PVC. Furthermore, although patients with diabetes, hypertension, hyperlipidemia, and those with higher $\mathrm{BMI}$, greater left ventricular end systolic diameter (LVESD) and interventricular septum thickness in diastole (IVSD) were more likely to experience frequent PVCs, these relationships were not statistically significant (Figure 1).

According to the results of Pearson correlation coefficient test, EFT thickness was significantly positively correlated with age $(\mathrm{r}=0.389, \mathrm{P}=0.005)$ and IVSD $(r=0.384, \mathrm{P}=0.006)$. Correlation of other parameters with EFT thickness are shown in table 2.
Based on obtained results in univariate analysis assessing the relation between EFT thickness and frequent PVCs, odds of greater EFT thickness were more in patients with PVC $>10$ per hour. However, the results were not statistically significant (OR: $1.14,95 \% \mathrm{CI}$; 0.62-2.07, $\mathrm{P}=0.669)$.

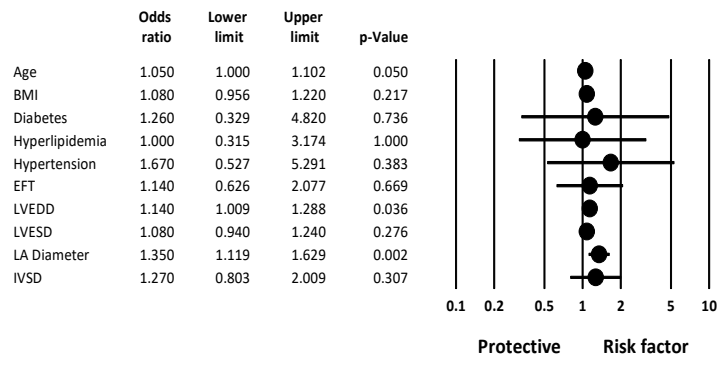

Figure 1: Demographic and echocardiographic parameters related to $\mathrm{PVC}$

Table 2: The relationship between EFT thicknesses with quantitative variables using pearson correlation coefficient

\begin{tabular}{|l|c|c|}
\hline & \multicolumn{2}{|c|}{ EFT Thickness } \\
\hline Age & r & P-value \\
\hline Body mass index & 0.389 & 0.005 \\
\hline Left ventricular end-diastolic dimension & 0.228 & 0.111 \\
\hline Left ventricular end-systolic dimension & 0.014 & 0.405 \\
\hline Left atrial Diameter & 0.247 & 0.925 \\
\hline Interventricular septal diameter & 0.384 & 0.083 \\
\hline
\end{tabular}

EFT=Epicardial fat tissue, $r=$ correlation

\section{DISCUSSION}

The present study seeks to investigate a possible relationship between EFT thickness and frequent PVCs in patients with no history of structural heart diseases.

The Current study revealed that patients with higher LVEDD and LA diameter were experiencing frequent PVCs. This finding is in consistency with a large and growing body of literature which previously have reported that recurrent PVCs can lead to a reduction in left ventricular function, and can induce adverse cardiac effects such as ventricular hypertrophy, cardiomyopathy, and chronic heart failure. ${ }^{15,16} \mathrm{LA}$ dilation and increased LVEDD reflect the severity of diastolic dysfunction and are prognostic for adverse cardiac outcome. ${ }^{17,18} \mathrm{LV}$ diastolic dysfunction indicates that there is an increment in resistance of ventricles and it is associated with the risk of developing $\mathrm{HF}$ and increased cardiovascular mortality. ${ }^{19}$

Moreover, another finding was that EFT thickness was significantly positively correlated with age. Similar to 
our findings, a study by Zhang et al. evaluating the association of epicardial adipose tissue with cardiovascular risk factors and coronary artery calcification showed that EFT thickness increases with age, height and weight. ${ }^{20}$ Also, Correlation analysis in the study by Shi et al. Showed that age was positively correlated with epicardial adipose tissue volume. ${ }^{22}$ As a person gets older, a gradual decrease in muscle tissue volume, as well as a gradual increase in body fat amount occurs. The body fat tissue in an adult increase by about $0.45 \mathrm{~kg}$ each year during the ages between $30-60$ years. This is mainly due to the increase in fat intake in addition to the increase in the amount of preadipocytes which are thenceforth differentiated into fat in order to proliferate adipose tissue. ${ }^{21}$ In contrast to earlier findings which demonstrated that higher BMI is associated with greater EFT thickness, ${ }^{22}$ however, no significant correlation between EFT thickness and BMI was detected in our study. This discrepancy could be attributed to the small sample size of the study, which limited investigating strong correlations and performing further analysis. In this research, there has been found a positive correlation between EFT thickness and some echocardiographic parameters, including LVEDD, LA diameter, and IVSD. Patients with greater LVEDD, IVSD, and LA enlargement had higher thickness in epicardial adipose tissue. However, the correlation of EFT with LVEDD and LA diameter was not statistically significant. These findings further support the idea of earlier inquiries which indicated that increased EFT thickness can result in structural changes such as LV mass, atrial and RV cavity size. ${ }^{23}$ EFT as a form of visceral fat tissue is known to be associated with a greater inflammatory state via releasing inflammatory cytokines such as tumor necrosis factor-alpha, interlukin1 and interlukin6, which can lead to structural and ultrastructural changes in the myocardium and electrical remodeling of the heart. All these changes may predispose the heart to arrhythmias such as ventricular extra-systoles. ${ }^{6,23}$ However, much uncertainty still exists about the relation between EFT thickness and PVCs. Surveys such as that conducted by Nabati et al. have shown a significant association between increased EFT thickness and some cardiovascular risk factors, including calcium deposits in the mitral valve annulus. ${ }^{24}$ Previous research findings into the relationship of EFT thickness and frequent ventricular premature beats in patients without structural heart diseases, performed by Kiris et al. have indicated that patients with frequent ventricular premature beats had increased EFT thickness in comparison to the control group and they concluded that EFT thickness was independently in association with ventricular premature beats. ${ }^{23}$ The results of the present study did not show any statistically significant correlation between EFT thickness and PVCs, however, with the increase in EFT thickness, the chance of experiencing frequent PVCs raised.

Finally, a number of important limitations need to be considered. First, the small sample size of the study. Second, the cross sectional design of the study limits casual relationships between EFT, PVCs and related factors. Third, EFT was assessed only by 2D transthoracic echocardiography, which is a reliable and practical method for assessing EFT, but no computed tomography or magnetic resonance imaging was performed. Therefore, it seems that designing larger cohort studies in addition to obtaining blood levels of inflammatory markers may be valuable in better investigating the mentioned relationships.

\section{CONCLUSION}

The results of this investigation showed that patients with frequent PVCs had significantly higher LVEDD and LA diameter, which provide additional evidence with respect to the association of recurrent PVCs and the risk of developing adverse cardiac effects such as cardiomyopathy and heart failure. In addition, another important finding of the study was that older patients and those with higher IVSD, LA diameter and LVEDD showed higher EFT thickness. Whilst this study did not confirm any statistically significant correlation between EFT thickness and frequent PVCs, obtained data showed that patients with higher EFT thickness were more likely to experience frequent PVCs.

\section{AUTHORS' CONTRIBUTION}

SFM and SS: Concept and design, data acquisition, interpretation, drafting, final approval, and agree to be accountable for all aspects of the work. AV, AS, SH, and MG: Data acquisition, interpretation, drafting, final approval and agree to be accountable for all aspects of the work.

Conflict of interest: Authors declared no conflict of interest.

\section{REFERENCES}

1. Panizo JG, Barra S, Mellor G, Heck P, Agarwal S. Premature ventricular complex-induced cardiomyopathy. Arrhythmia \& electrophysiol Rev. 2018;7(2):128.

2. Ahn M-S. Current concepts of premature ventricular contractions. J Lifestyle Med. 2013;3(1):26-33.

3. Frigy A, Csiki E, Caraşca C, Szabó IA, Moga V-D. Autonomic influences related to frequent ventricular premature beats in patients without structural heart disease. Medicine (Baltimore). 2018;97(28).

4. Winkens R, Höppener P, Kragten J, Verburg M, Crebolder H. Are premature ventricular contractions always harmless? Eur J Gen Pract. 2014;20(2):134-8.

5. Emkanjoo Z, Mollazadeh R, Alizadeh A, Kheirkhah J, Mohammadi Z, Khalili M, et al. Electrocardiographic (ECG) clues 
to differentiate idiopathic right ventricular outflow tract tachycardia (RVOTT) from arrhythmogenic right ventricular cardiomyopathy (ARVC). Indian Heart J. 2014;66(6):607-11.

6. Kanat S, Karaduman BD, Tütüncü A, Tenekecioğlu E, Mutluer FO, Bayram NA. Effect of Echocardiographic Epicardial Adipose Tissue Thickness on Success Rates of Premature Ventricular Contraction Ablation. Balkan Med J. 2019;36(6):324.

7. Chen T, Koene R, Benditt DG, Lü F. Ventricular ectopy in patients with left ventricular dysfunction: Should it be treated? J Card Fail. 2013;19(1):40-9.

8. Tok D, Kadife İ, Turak O, Ozcan F, Başar N, Çağlı K, et al. Increased epicardial fat thickness is associated with low grade systemic inflammation in metabolic syndrome. Turk Kardiyol Dern Ars. 2012;40(8):690-5.

9. Gaeta M, Bandera F, Tassinari F, Capasso L, Cargnelutti M, Pelissero G, et al. Is epicardial fat depot associated with atrial fibrillation? A systematic review and meta-analysis. Europace. 2017;19(5):747-52.

10. Acet H, Ertaş F, Akıl MA, Oylumlu M, Polat N, Yıldız A, et al. New inflammatory predictors for non-valvular atrial fibrillation: echocardiographic epicardial fat thickness and neutrophil to lymphocyte ratio. Int J Cardiovasc Imaging. 2014;30(1):81-9.

11. Shamloo AS, Schoene K, Stauber A, Darma A, Dagres N, Dinov $\mathrm{B}$, et al. Epicardial adipose tissue thickness as an independent predictor of ventricular tachycardia recurrence following ablation. Heart Rhythm. 2019;16(10):1492-8.

12. White E, Saint DA. Increased mechanically-induced ectopy in the hypertrophied heart. Prog Biophys Mol Biol. 2012;110(2-3):3319.

13. Chistiakov DA, Grechko AV, Myasoedova VA, Melnichenko AA, Orekhov AN. Impact of the cardiovascular system-associated adipose tissue on atherosclerotic pathology. Atherosclerosis. 2017;263:361-8.

14. Duncan DJ, Yang Z, Hopkins PM, Steele DS, Harrison SM. TNF$\alpha$ and IL-1 $\beta$ increase $\mathrm{Ca} 2+$ leak from the sarcoplasmic reticulum and susceptibility to arrhythmia in rat ventricular myocytes. Cell calcium. 2010;47(4):378-86.

\section{Address for Correspondence:}

Dr. Mahboobeh Gholipour, Cardiovascular Diseases Research Center, Department of Cardiology, Heshmat Hospital School of Medicine, Guilan University of Medical Sciences, Rasht, Iran.

Email: m.gholipour.md@gmail.com
15. Baser K, Bas HD, LaBounty T, Yokokawa M, Good E, Latchamsetty R, et al. Recurrence of PVCs in patients with PVCinduced cardiomyopathy. Heart Rhythm. 2015;12(7):1519-23.

16. Yokokawa M, Good E, Crawford T, Chugh A, Pelosi Jr F, Latchamsetty R, et al. Recovery from left ventricular dysfunction after ablation of frequent premature ventricular complexes. Heart Rhythm. 2013;10(2):172-5.

17. Patel DA, Lavie CJ, Milani RV, Shah S, Gilliland Y. Clinical implications of left atrial enlargement: a review. Ochsner J. 2009;9(4):191-6.

18. Kajimoto K, Minami Y, Otsubo S, Sato N, Registry IotADHFS. Sex differences in left ventricular cavity dilation and outcomes in acute heart failure patients with left ventricular systolic dysfunction. Can J Cardiol. 2018;34(4):477-84.

19. Topuz M, Dogan A. The effect of epicardial adipose tissue thickness on left ventricular diastolic functions in patients with normal coronary arteries. Kardiologia Polska. 2017;75(3):196203.

20. Zhang H, Meng X, Wang W, Zhang K, Qi Y, An S, et al. Association of epicardial adipose tissue with cardiovascular risk factors and coronary artery calcification in the community residents. Zhonghua Xin Xue Guan Bing Za Zhi. 2018;46(5):3649.

21. Shi K, Qi L, Mao D, Chen Y, Qian J, Sun Y, et al. Impact of age on epicardial and pericoronary adipose tissue volume. Eur Rev Med Pharmacol Sci. 2015;19(17):3257-65.

22. Wang M, Zhao L, Liang H, Zhang C, Guan L, Li M. A new measurement site for echocardiographic epicardial adipose tissue thickness and its value in predicting metabolic syndrome. Advances Clin Experiment Med. 2019;28(10):1403-8.

23. Kırıș A, Turan OE, Kırış G, İlter A, Öztürk M, Aydın M, et al. The relationship between epicardial fat tissue thickness and frequent ventricular premature beats. Kardiologia polska. 2015;73(7):52732

24. Nabati M, Salehi A, Hatami G, Dabirian M, Yazdani J, Parsaee H. Epicardial adipose tissue and its association with cardiovascular risk factors and mitral annular calcium deposits. Ultrasound. $2019 ; 27(4): 217-24$ 